\title{
Stress Analysis of Centrifugal Fan Impellers
}

\author{
Dr. Muhamed H. Abood* Ayad A. Ghaidan** Mahmood H. Ali** \\ * Karbala University / College of Engineering $\quad$ **niversity of Kirkuk / College of Engineering
}

\begin{abstract}
This paper deals with the determination of stress distribution for a centrifugal fan consists of back sheet, ten airfoil blades and shroud by using Finite element Packaged program (NSTRAN). The results showed that the blade trailing edge and the shroud plate have a maximum Von Mises stress which exceeds the yield point of the materials. Thickness effect of each part on the stress distribution in the impeller was determined with fixing the other parts thickness. The result showed that the optimum thickness of the plate is $(11 \mathrm{~mm})$, for shroud is $(9 \mathrm{~mm})$ for blade is $(3 \mathrm{~mm})$, for back sheet plate is $(11 \mathrm{~mm})$ for shroud stiffener is $(3 \mathrm{~mm})$. the overall results pointed out that the shroud plate is a critical part and has a large effect on the stress distribution than other parts.
\end{abstract}

Keywords: Centrifugal Fan, Von Mises stress, Finite Element, NASTRAN

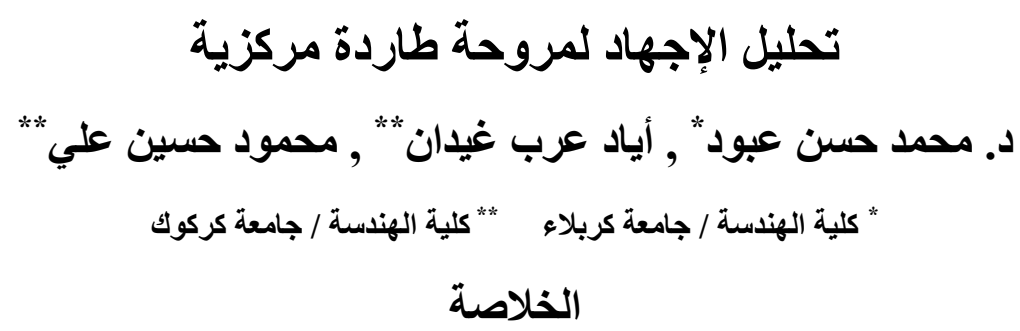

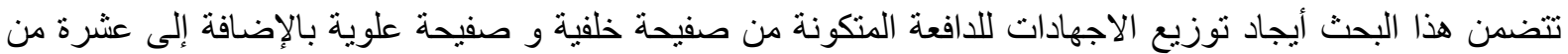

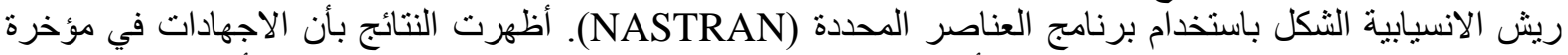

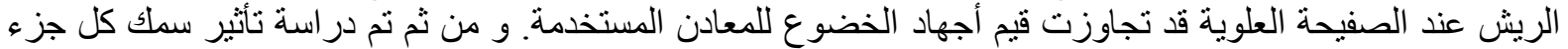

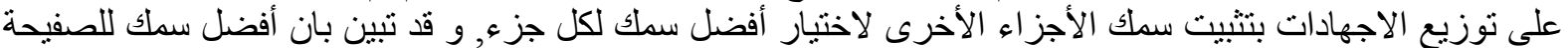

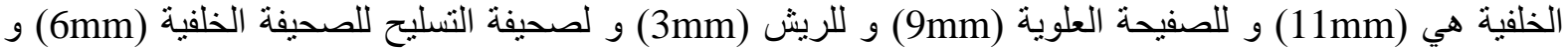

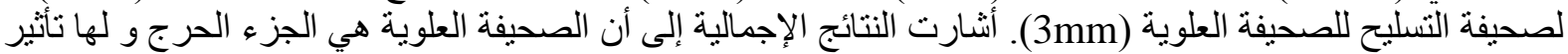

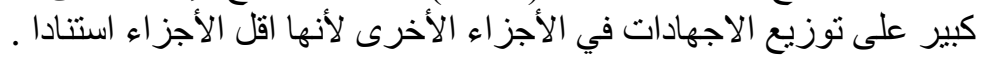




\section{Introduction:}

There are few methods and references that deal with stress analysis of centrifugal fan impeller. For a long time the conventional methods proposed by Dnath (1912), Hearle (1918) (based on stress in rotating disk) and Tumarkin (1944) were used to analyze the stresses in the centrifugal fans (reported by [1,2]).

The two methods involve a separate treatment of each part from another, the back sheet (main disk) and the cone sheet (shroud) are considered in the analysis as an isolated rotary disk. The blade loading is added at appropriate radii, divided as a percentage ratio between these two parts.

The third method assumes the rotor to be symmetrical about the normal plane of the rotation axis and the blade does not participate in the stresses transmission, and their masses are uniformly distributed over the angular coordinate.

These methods are the base of all designs of centrifugal impellers before the great developments in digital computers, which made possible to solve large numbers of equations formed by the numerical methods such as finite element and finite difference.

Dahlgrn et al., 1985[3], employed the finite element stress analysis and a model analysis in conjugation with strain gauge measurement to evaluate a failure of large centrifugal fan. They use a fan failed in the Electricity Supply Commission (ESCOM) power plant in south Africa as a base of their work .

Schiffer, 1986 [4] analyzed a radial flow turbine impeller using (Ansys) program to determine centrifugal stresses and the mode frequency solution. A variety of the modeling procedure for the hub and vans are described with sample of solution output for the original and modified design of the vans, which are modeled using plate finite element. The analyzed impeller consists of (17) integral vans with curved surface of variable thickness. The most important conclusions drown by the author is that the maximum stress location is at the hub bore. The maximum stress for the vans correlates well with the observed crack in the failed vans. The modified design impeller showed a substantial stress reduction about $84 \%$ of the original stress.

Irrtier, 1988 [5], presented experimental and numerical results of the centrifugal stresses and vibration of rotating radial impeller consisting of ten sheet plate blade welded between base (back) and cover (shroud) plate. He presented stress distribution due to rotation at the top edges of blades and the welding seams between the blade and other two parts and its deformation, natural frequencies as a function of rotational speed, mode shapes, resonance excitation and frequencies.

Lei Zhang, 2011[6] presented experimental and numerical results plate on G4-75 centrifugal fan as a reference model, A practical parametric software plat form was developed with VB programming language and fluent software, the results show that the total pressure and efficiency of fans will be ideal when the blade angle take 45 degree, which is the design value, However, the best impeller width gotten from numerical simulation is smaller than the design value.

Ray et al., 1989 [7], made the differential equations produced by Schilhansl in a nondimensional form and presented a computer method for optimizing stress distribution in a radial flow impeller according to given constrains that specified for required design. The 
results obtained from three worked examples by the author showed a same rotor profile with that obtained from other methods, with some irregularities in cross sectional areas owing to discritized nature of the analytical scheme that can be improved by taking a large number of segments in the analysis.

\section{Finite element Modeling:}

The problem of stress analysis in a centrifugal fan impeller can be accurately carried out through the use of finite element method, which is a powerful tool in modeling a fan structure and predicting its response [1,2].

Finite element is methods that are used for a wide range of engineering problems, which becomes a powerful tool for the numerical solution with the advances in computer technology.

The variation of the field variable (displacement, temperature, velocity etc) in the element is not known, so, it is approximated by a simple function relationship (interpolation models, usually polynomial function) and defined in terms of the unknown values at the nodes. The general displacement function has a matrix form as [7].

$\{U\}=[N] .\{a\}$

$\{a\}$ is the assumed displacement vector constants.

$[N]$ is the shape function matrix.

$\{U\}$ is the nodal displacement vector.

The partial derivatives of the displacement $\mathrm{u}, \mathrm{v}$, and $\mathrm{w}$ in direction $\mathrm{x}, \mathrm{y}$, and $\mathrm{z}$ respectively, can express the strain in the deformed structure, these equations have a matrix form [7].

$\{\varepsilon\}=[X]\{U\}$

Where:

$\{\varepsilon\}$ is the strain vector

$[X]$ is a transforming operator matrix, which contain partial derivatives.

Since the relationships between the elastic strains and stresses are related by means of the Hook's law, after solving for stresses it can be written in matrix form as [7].

$\{\sigma\}=[E]\{\varepsilon\}$

Where

$\{\sigma\}$ is the stress vector

$[E]$ is the elasticity matrix

$\{\varepsilon\}$ is the strain vector

For linearly elastic structure, the strain energy $S$ of an element in the structure can be represented in terms of strain and stresses as

$s=\frac{1}{2} \int\{\varepsilon\}^{T} \sigma d V$ 
$\mathrm{V}$ indicates that the integral is over the volume.

The successive substitution of equations (1), (2) and (3) then substituting the results in equation (4) yields an expression for element strain energy as:

$S=\frac{1}{2} \int\{U\}^{T}[X]^{T}[E][X]\{U\} d V$

Or

$S=\frac{1}{2}[X]^{T}[K]\{U\}$

Where $[K]$ is Known as the stiffness matrix of the element and is given by:

$K=\int X^{T}[E][X] d V$

The potential energy $(\mathrm{P})$ of the structure or body with external forces $(\mathrm{F})$ can be expressed as [21].

$P=S-\{U\}^{T}\{F\}$

The static equilibrium configuration of the structure requires a minimization of potential energy with respect to each degree of freedom, the final form of the equation is [8].

$\{F\}=[K]\{U\}$

The system of equations (8) is the general form of any element type and is the starting point of solution for elements that represents the structure which gives the displacement components for each node that can be used in successive substitution in the previous equations to get desired parameters such as stress, strain, force and any other.

In this paper a finite element modeling and discretization of two centrifugal fan impellers presented. First one that presently used named as (model I) and the other is suggested design named as (model II). The impeller shapes and dimensions for ( model I) is shown in Fig. (1). the back plate has an outer diameter of $(1800 \mathrm{~mm})$ and $(150 \mathrm{~mm})$ in inner diameter with thickness of $(12 \mathrm{~mm})$.
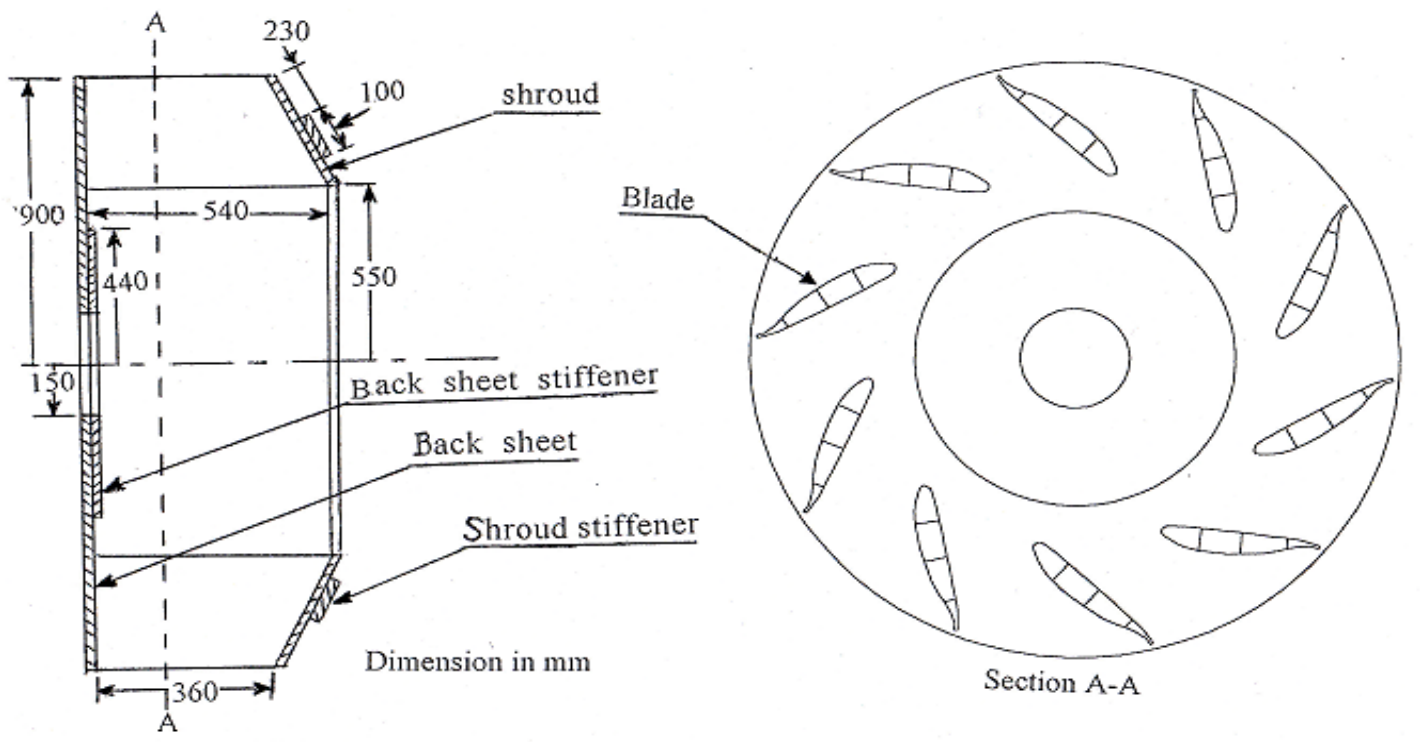

Fig.(1) Drawing of the Impeller in a company 
The blade width is $(540 \mathrm{~mm})$ at the inlet and $(360 \mathrm{~mm})$ at the outlet the plate thickness is $(4 \mathrm{~mm})$ with three stiffeners fitted inside it to increase its rigidity. The shroud thickness is $(6 \mathrm{~mm})$ with an inside diameter of $(540 \mathrm{~mm})$ and outside diameter of $(1800 \mathrm{~mm})$. ( Model II) was shown in Fig.(2) and its dimensions as table (1).

Half of each impeller is considered in the modeling and analysis because the other half's action can be considered through the state of anti symmetry that occurs in the impeller by applying a required boundary condition at the cutting edge. Same loading condition and boundary conditions are used for these two models
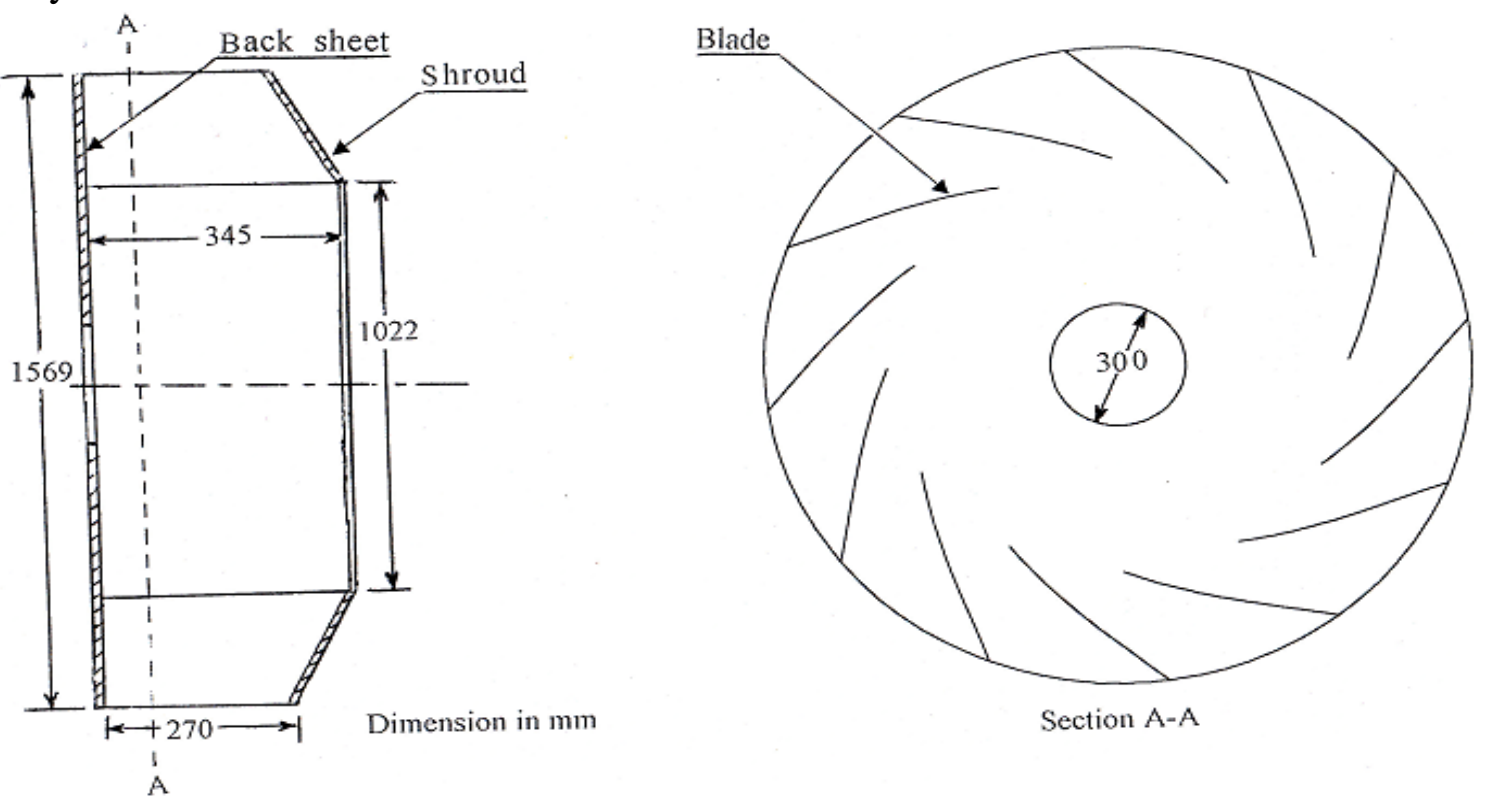

Fig.(2)Drawing of suggested Impeller

Table(1) Dimension of Suggested Impeller

\begin{tabular}{|c|c|c|c|c|}
\hline $\mathrm{d} 1(\mathrm{~mm})$ & $\mathrm{d} 2(\mathrm{~mm})$ & $\mathrm{b} 1(\mathrm{~mm})$ & $\mathrm{b} 2(\mathrm{~mm})$ & $\mathrm{Z}$ \\
\hline 1022 & 1569 & 345 & 270 & 12 \\
\hline
\end{tabular}

\subsection{Loading Condition:}

There are three types of loading that actually act on the centrifugal fan impeller. The first one is the centrifugal force because of impeller rotation that resulted in centrifugal acceleration of the impeller body. The second is that resulted from thermal expansion caused by temperature rising. The last one is the aerodynamic force from pressure conversion between the blade and the air $[1,2]$.

In the present work, the analysis for each model was performed under the centrifugal force only. The effect of aerodynamic force is ignored because this force is very small as compared to the large centrifugal force. The thermal load is ignored because the air is consumed from the atmosphere and supplied to the furnace burner without causing temperature rise and hence no thermal effect was considered.

In the finite element method the distributed loads are converted to nodal forces. This was done through lumped load system in which the distributed loads are calculated at the centroid 
of the element and then divided equally to the surrounding nodes [8]. So that the centrifugal force calculated at the centroid of the element from Newton's law:

$F_{e}=\rho V r_{c} \omega^{2}$

Where

$F_{e}$ : element force.

$V$ : Volume of the element.

$r_{c}$ : element centroid from rotation axis.

$\omega$ : rotational velocity of the body.

\subsection{Boundary condition and material property:}

The impeller is fixed at the edge against any rotation and translation in each of the three directions $\mathrm{x}, \mathrm{y}$, and $\mathrm{z}$ to represent the rigid connection between the impeller and its supporting shaft.

In order to find the boundary conditions that required for analyzing one blade structure, for this condition the freedom of the cutting edge is chosen as follows

$\delta_{r}=0, \quad \delta_{\theta}=1, \quad \delta_{z}=0$

$\theta_{r}=1, \quad \theta_{\theta}=0, \quad \theta_{z}=0$

$\theta_{r}, \theta_{\theta}, \theta_{z}$ are the Nodal rotations of the element.

$\delta$ is the deflection.

Where:

1: restricted deformation

0 : free deformation

The material property of impeller that presently used in the (N.G.I) company, is obtained by a tensile test of standard specimens according to Japanese Industrial Standard (JIS Z2201 1980) cut from the impeller and tested using (WAW 1000) computerized tensile test machine. The Young modulus of elasticity and Poisson's ration are obtained equal to (207 Mpa and 0.3) respectively according to the property specified for carbon steel alloys in reference [10]. Shear modulus is calculated from the relation $[G=E / 2(1+v)]$ and is equal to $(79.6 \mathrm{Mpa})$. Table (2) show the material property obtained from test.

Table(2) Material Property Obtained From Tests

\begin{tabular}{|c|c|c|c|c|c|c|}
\hline Element & Test No. & бу (Мpa) & $\begin{array}{l}\text { Average } \\
\text { бy (Mpa) }\end{array}$ & $\begin{array}{c}\sigma \mathrm{u} \\
(\mathrm{Mpa})\end{array}$ & $\begin{array}{l}\text { Average } \\
\text { ou (Mpa) }\end{array}$ & $\mathrm{p}\left(\mathrm{kg} / \mathrm{m}^{3}\right)$ \\
\hline \multirow{2}{*}{ Bake plate } & 1 & 475 & \multirow[t]{2}{*}{462.5} & 565 & \multirow[t]{2}{*}{560} & \multirow[t]{2}{*}{7900} \\
\hline & 2 & 450 & & 555 & & \\
\hline \multirow{3}{*}{$\begin{array}{l}\text { Shroud } \\
\text { plate }\end{array}$} & 1 & 470 & \multirow[t]{3}{*}{470} & 585 & \multirow[t]{3}{*}{557} & \multirow[t]{3}{*}{7450} \\
\hline & 2 & 480 & & 540 & & \\
\hline & 3 & 460 & & 545 & & \\
\hline \multirow[t]{2}{*}{ Blade plate } & 1 & 470 & \multirow[t]{2}{*}{460} & 510 & \multirow[t]{2}{*}{530} & \multirow[t]{2}{*}{7700} \\
\hline & 2 & 450 & & 550 & & \\
\hline
\end{tabular}

$\sigma y$ : yield point stress, $\sigma u$ : ultimate point stress, P: mass density 


\section{3: Element Description:}

Half of each impeller is considered in modeling and analysis because the other half's action can be considered through the state of antisymmetry that occur in the impeller by applying a required boundary condition at the cutting edge.

For Back Plat represented by three node triangle plate element, with thickness of $(12 \mathrm{~mm})$, the bending and membrane behaviors are considered. The element has total of (18) degrees of freedom, i. e. three displacements and three rotations per node.

The blades are represented by a four-node shear panel element connected between the shroud and the back plate. The blades and other two parts (shroud and back plate) are shared by nodes to represent the welding between them. Appropriate element size is considered at the curved region for the best representation and avoidance of hard curvature.

The shroud and its reinforcement plate are also represented by a three-node triangular plate element. The location of the nodes and elements are projection of the base plate elements. The welding between the shroud and reinforcement plate is represented by shear panel element.

\section{Results and Discussion:}

The analysis were carried out for the original fan impeller which has back sheet thickness of $12 \mathrm{~mm}$, shroud $6 \mathrm{~mm}$, blade $4 \mathrm{~mm}$, back sheet stiffener $8 \mathrm{~mm}$ and $4 \mathrm{~mm}$ for the shroud stiffener. The deformed shape of the impeller is shown in Fig. (3). it can be seen that the shroud tends to deflect inward in the space between any two adjacent blades at the outer radius with a maximum value of $(8.46 \mathrm{~mm})$, while it deflects outward at the inner radius. No deflections were observed near stiffener zone because this region requires higher stresses to be deflected as it more rigid than other zone. These deflections are mainly due to bending induced stresses.

The deflection of the back sheet is also a maximum at mid distance between the blades with a value of (5.82) which is smaller than deflection of the shroud.

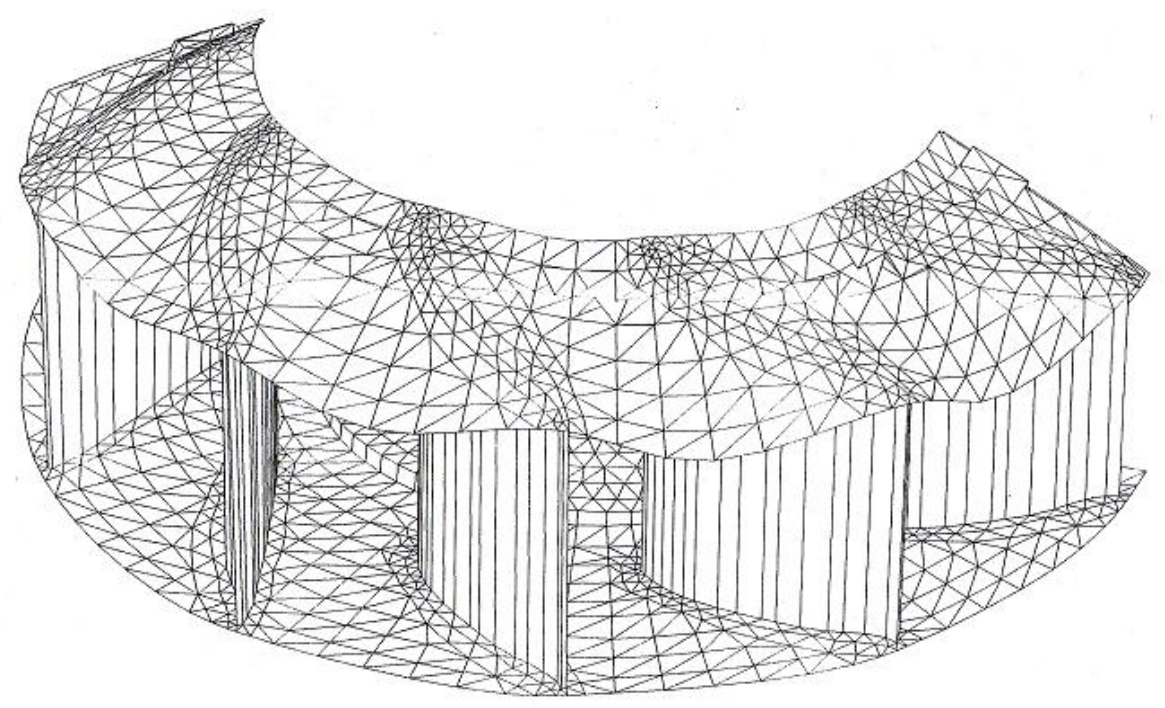

Fig(3). Deformation of Fan Impeller(Model I) 
Figure (4) shows the value of maximum the Von Mises stress for each part as related to the shroud thickness. According to this figure the optimum shroud thickness is $(9 \mathrm{~mm})$, which lead to a good results as maintaining the stress in each parts to be than the yield point of the materials.

Figure (5) shows the stress distribution in the with variation of blade thickness for range ( 3 to 6) $\mathrm{mm}$, from this figure, the optimum blade thickness is obtained to be $(3 \mathrm{~mm})$ thus resulting in minimum stress in all parts.

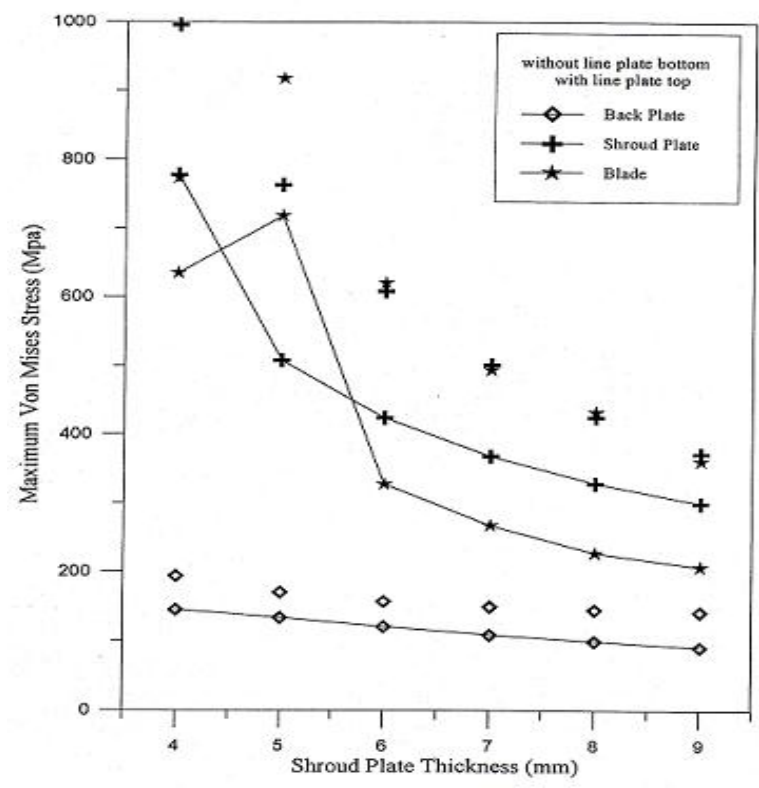

Fig(4). Maximum Von Mises stress variation with shroud plate thickness.

The stress variation with back plate thickness is shown in the figure (6), for a range of (10 to 14)mm. The optimum thickness of the back sheet is chosen to be $(11 \mathrm{~mm})$, taking into consideration the weight of the parts.

The effect of the back plate stiffener thickness on the stress distribution in other parts is presented in the figure (7). The optimum thickness of this part is chosen to be $(6 \mathrm{~mm})$ in order to decrease its mass.

The analysis was carried out for effect of shroud plate stiffener thickness from (3to 6) $\mathrm{mm}$, and the results are shown in figure (8) when this part was removed it was observed that the stress in the back sheet increases about $18 \%$ for bottom face and $40.5 \%$ for top face.

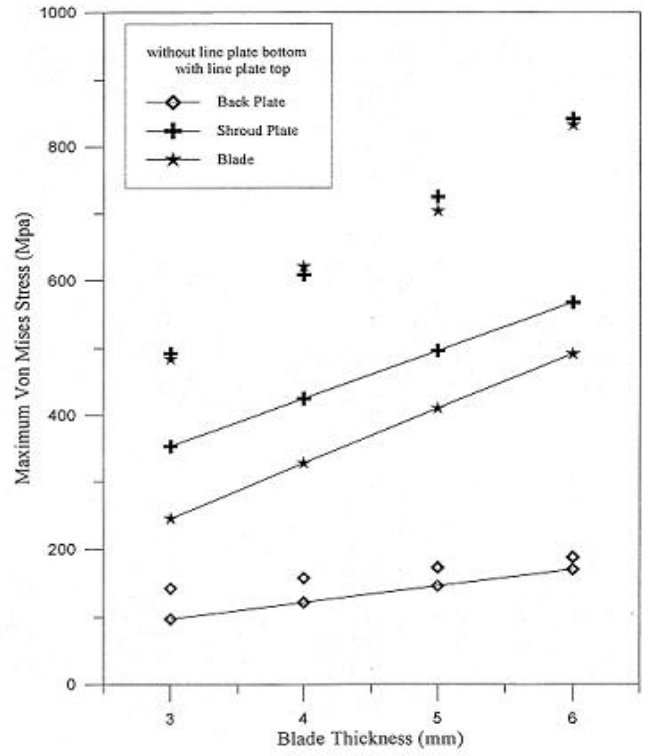

Fig.(5) Maximum Von Mises stress variation with blade plate thickness.

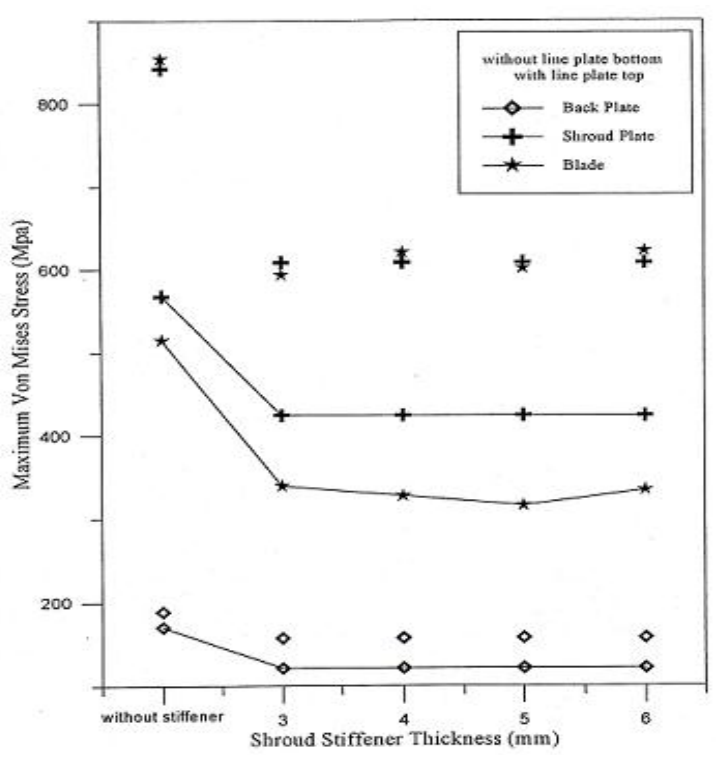

Fig.(8) Maximum Von Mises stress variation with shroud plate stiffener thickness. 
The optimum thickness of this part was considered to be $(5 \mathrm{~mm})$, which minimizes the maximum Von Mises stress for some parts, while maintaining it constant for others.

\section{Conclusion:}

The maximum deflection in the shroud and back sheet appears to be at mid span between the blades, this deflection is very dangerous when vibration excitation is exist, thus leading to failure, and the stress also in the shroud is higher than other parts so that the shroud is the

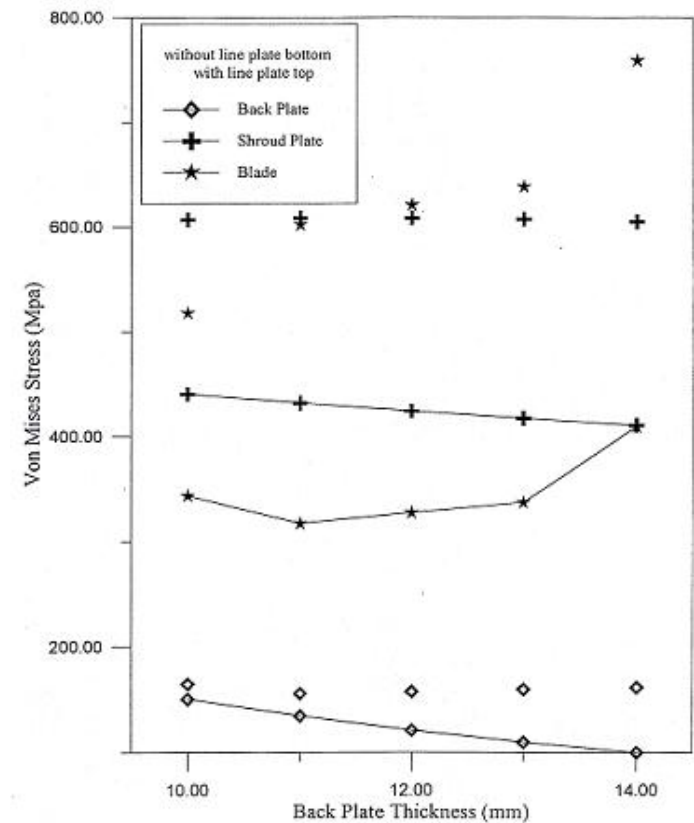

Fig.(6) Maximum Von Mises stress variation with back plate thickness

most critical part in the impeller.

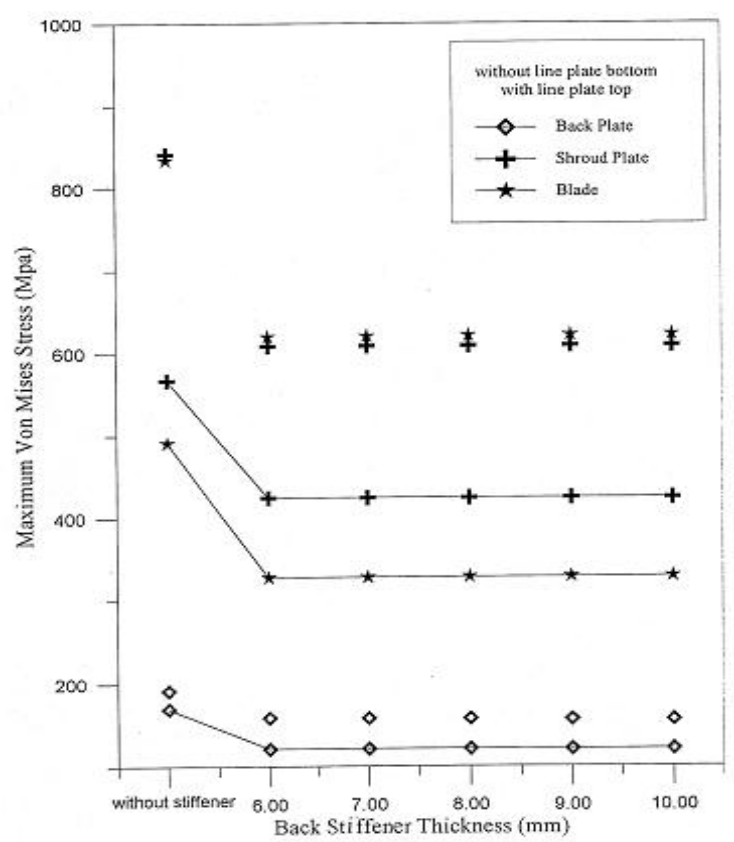

Fig.(7) Maximum Von Mises stress variation with hack nlate stiffener thickness

From the stress distribution obtained for the original design, different materials can be applied thus sustaining the induced stresses, without changing any of the geometrical dimensions.

The thickness of the stiffener of both back sheet and shroud has no effect on the stress in other parts, while their absence rise the stress in these parts by a range $12 \%$ to $35 \%$ for various parts, so that the thickness of shroud stiffener is suggested to be $(3 \mathrm{~mm})$ and back sheet stiffener $(6 \mathrm{~mm})$ that maintain the mass of these parts at lower values. The back sheet and its stiffener must be forged or machined as one part to avoid welding defect and increase the rigidity of the part. 


\section{References:}

1. Bell, R. and Benham, P. P. " Theoretical and Experimental Stress Analysis of Centrifugal Fan Impellers", Journal of Strain Analysis, Vol, 13 No 3 1978 P 141-147.

2. Patton, R. G. " Stresses in Centrifugal Fan Impellers" Proc Instn Mech Engrs Vol 187 No 281973 P 309-315.

3. Dahlgren, C. A. et, al. "Fatigue Failure of a Large Industrial Fan", Fracture and Fracture Mechanics, Cas Studes, Pergamon Press 1985 P 137-146.

4. Schiffer, D. " $3 \mathrm{D}$ Modeling of an Expander Impeller" the Finite Element User's Conference Rochester 1986, DRESSER-RAND Technical Publications 1987.

5. Irretier, H. " Numerical and Experimental Investigation of Stresses and Vibrations of Rotating Radial Impeller', Institute for Mechanics, Gh Kassel University 1988.

6. Lei Z. and S.." Numerical Study for centrifugal Fan Based on Parametric Method", Applied Mechanics and Materials,Vols-55-57,(2011), PP892-897.

7. Ray, G. S. “ Optimum Design of Radial Flow Impellers", Proc Instn Mech. Engrs 1989 Vol. 203 Part A Journal of Power Engineering.

8. Bathe, K. J. " Finite Element Procedures", Prentic Hall International Incorporation, 1996.

9. Rao, S. S. “ The Finite Element Method in Engineering”, Program on Press 1982.

10. Nortin, Robert L. “Machine Design” Prentice-Hall 1998. 